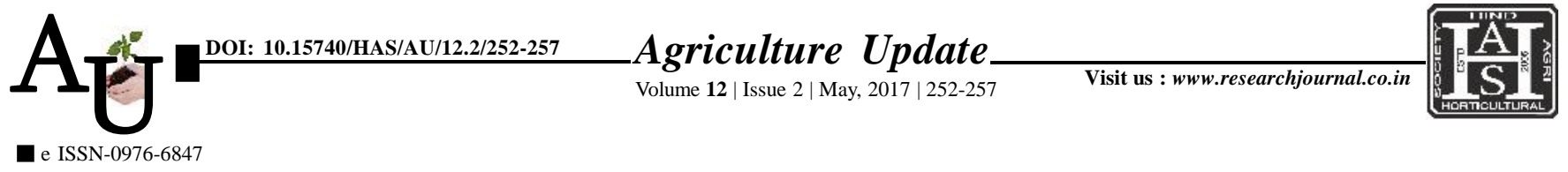

\title{
Research Article: Modelling and forecasting of tur production in India using ARIMA model
}

\section{PREMA BORKAR AND V.M. BODADE}

Article Chronicle: Received : 07.03.2017;

Revised : 22.03.2017;

Accepted :

04.04.2017
SUMMARY : The paper describes an empirical study of modeling and forecasting time series data of tur production in India. Yearly tur production data for the period of 1950-1951 to 2014-2015 of India were analyzed by time-series methods. Autocorrelation and partial autocorrelation functions were calculated for the data. The Box Jenkins ARIMA methodology has been used for forecasting. The diagnostic checking has shown that ARIMA $(1,1,1)$ is appropriate. The forecasts from 2015-2016 to 2024-2025 were calculated based on the selected model. The forecasting power of autoregressive integrated moving average model was used to forecast tur production for ten leading years. These forecasts would be helpful for the policy makers to foresee ahead of time the future requirements of tur seed, import and/or export and adopt appropriate measures in this regard.

How to cite this article : Borkar, Prema and Bodade, V.M. (2017). Modelling and forecasting of tur production in India using ARIMA model. Agric. Update, 12(2): 252-257; DOI : 10.15740/HAS/AU/12.2/252-257.

\section{KEY WoRDS:}

ACF - autocorrelation function, ARIMA autoregressive integrated moving average, PACF - partial autocorrelation function, Tur 